Tropical Journal of Applied Natural Sciences

Trop. J. Appl. Nat. Sci., 2(2): 15-25 (2018)

ISSN: 2449-2043

https://doi.org/10.25240/TJANS.2018.2.2.03

\title{
Uniqueness of Optimal Control of Nonlinear Infinite Neutral Functional Differential Systems with Distributed Delays in the Control
}

\author{
Paul Anaetodike Oraekie \\ Department of Mathematics, \\ Chukwuemeka Odumegwu Ojukwu University, Uli- Campus \\ Nigeria, West Africa \\ E-mail: drsirpauloraekie@gmail.com; Phone no.: +2347031982483
}

\begin{abstract}
In this paper, Nonlinear Infinitely Neutral Functional Differential Systems with Distributed Delays in the Control was presented for controllability analysis. The system was linearized using Chukwu (1992)-like arguments. After linearization, we obtained the mild solution of the system using variation of constant formula and the Unsymmetric Fubuni Theorem to establish the results. The set functions (reachable set, target set, attainable set and controllability grammian) upon which our study hinged were extracted. Necessary and sufficient conditions for the establishment of the uniqueness of the system were derived. Use was made of some controllability standards to establish results. The establishment of the uniqueness of the optimal control provided a new approach for the proof of the existence of an optimal control of any dynamical control system.

Keywords:

Uniqueness

Optimal control

Admissible control

Reachable set

Attainable set.
\end{abstract}

Original Research Article

Received: $05^{\text {th }}$ Dec., 2017

Accepted: $07^{\text {th }}$ Feb., 2018

Published: $14^{\text {th }}$ Mar., 2018

\section{INTRODUCTION}

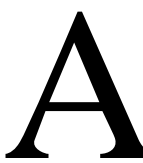

neutral functional differential equation is given as a differential equation depending on past and present values which involve derivatives with delays (see Banks and Kent (1972)). This means that the derivatives of the functional

difference operator $D(t, x)$ is expressed in terms of the past and present states or the unknown functions. Neutral equations have been found to have great importance in various applications in natural sciences, technology and electrodynamics (see Balachandran and Dauer (1996); (2002)). In his paper “Linear Systems with Delayed Control” by Artstein (1982), the use of neutral equations in the study of electrical networks containing lossless transmission lines is made evident. Chukwu (2001) modeled a neutral dynamics to represent the rate of growth of the world economy.

The existence and uniqueness conditions for neutral functional differential equation have been presented (see Anichini et al. (1986); Balachandran (1992)). Research on neutral systems has extended to finding necessary and sufficient conditions for the asymptotic behavior of solutions of such equations. Balachandran and Leelamani (2006), studies stability of infinite neutral systems.

We hope to investigate the controllability of nonlinear infinite Neutral Differential Systems with distributed Delays in Control of the form: 


$$
\begin{array}{ll} 
& \frac{d}{d t}\left[D\left(t, x_{t}\right)\right]=L\left(t, x_{t}\right) x_{t}+\int_{-\infty}^{0} \mathrm{~A}(\theta) x(t+\theta) d \theta+\int_{-h}^{o}\left[d_{\theta} H(t, \theta)\right] u(t+\theta) \\
& +f\left(t, x_{t}\right) \\
x_{t_{0}}=\phi &
\end{array}
$$

(a nonlinear infinite neutral system).

Our principal objective in this paper is to obtain necessary and sufficient conditions for controllability, and existence of an optimal control for the system (1.1) above. It is known from Onwuatu (1993) that, if a system is relatively controllable, then optimal control is unique and bang-bang. In the light of this, we shall consider the nonlinear infinite space of Neutral Differential Systems with Distributed Delays in Control of the form: system (1.1) above. The above system (1.1) will be investigated for existence and uniqueness of optimal control, to achieve this, we shall begin by considering the relative controllability of the system.

\section{NOT ATION AND PRELIMINARIES}

Let $n$ be a positive integer and $E=(-\infty, \infty)$ be the real line. Denote by $E^{n}$ the space of real $n$-tuples called the Euclidean space with normdenoted by |.|. $x\left(t_{0}\right)=\phi=x_{0}$ is initial condition (function), where $x \in E^{n}$ is the state space and $u \in E^{m}$ is the control function, $(H(t, \theta)$ is an nxm matrix continuous at $t$ and of bounded variation in $\theta$ on $[-h, 0] ; h>0$ for each $t \in\left[t_{0}, t_{1}\right] ; t_{1}>t_{0}$. The $n x n$ matrix $A(t)$ is continuous in its argument if $J=\left[t_{0}, t_{1}\right]$ is any interval of $E, L_{2}$ is the Lebesque space of square integrable functions from $J$ to $E^{n}$ written in full as $L_{2}\left(\left[t_{0}, t_{1}\right], E^{n}\right)$.

Let $h>0$ be a positive real number and let $C\left([-h, 0], E^{n}\right)$ be a Banach space of continuous functions with the norm of uniform convergence defined by

$$
\|\phi\|=\sup |\phi(s)| ;-h \leq s \leq 0, \text { for } \phi \in C\left([-h, 0], E^{n}\right)
$$

If $x$ is a function from $[-h, 0]$ to $E^{n}$, then $x_{t}$ is a function defined on the delay interval $[-h, 0]$ given as:

$$
\mathrm{x}_{\mathrm{t}}(s)=x(t+s) ; s \in[-h, 0], t \in[0, \infty) .
$$

Consider the system 1.1) - the nonlinear infinite neutral system with distributed delays in control,

$$
\begin{gathered}
\frac{d}{d t}\left[D\left(t, x_{t}\right)\right]=L\left(t, x_{t}\right) x_{t}+\int_{0}^{\infty} A(\theta) x(t+\theta) d \theta \\
+\int_{-h}^{0}\left[d_{\theta} H(t, \theta)\right] u(t+\theta)+f\left(t, x_{t}\right)
\end{gathered}
$$

Where, $\quad L\left(t, x_{t}\right)=\sum_{k=0}^{\infty} A_{k} x\left(t-w_{k}\right)+\int_{-\infty}^{0} A(\theta) x(t+\theta) d \theta$.

$$
\begin{aligned}
& L\left(t, x_{t}\right) x_{t}=\int_{-\mathrm{h}}^{0} \mathrm{~d}_{\theta} \mathrm{\eta}(t, \theta, x(t+\theta)) x(t+\theta) \\
& \qquad \begin{array}{r}
\mathrm{g}(t, \theta, \phi, \Psi) \geq 0, \text { for } \theta \geq 0 \text { and } \phi, \Psi \in C . \\
\mathrm{\eta}(t, \theta, \phi, \Psi)=\mathrm{\eta}(t, \theta, \phi, \Psi), \text { for } \theta<-h .
\end{array}
\end{aligned}
$$

$\mathrm{\eta}(t, \theta, \phi, \Psi)$ is a continuous matrix function of bounded variation in $\theta \in[-h, 0]$, 


$$
\operatorname{vary} \mathrm{\eta}(t) \leq m(t), m(t) \in L_{1} .
$$

where $L_{1}$ is the space of integrable functions.

Let $\Omega$ be an open subset of ExC and $D$ and $L$ be bounded linear operators defined on

ExC into $E^{n}$.

$$
\begin{gathered}
D\left(t, x_{t}\right)=x(t) g\left(t, x_{t}\right), \\
\text { where, } g(t, \phi)=\sum_{n=0}^{\infty} A_{n}(t) \phi\left(-w_{n}(t)+\int_{-h}^{0} A(t, s) \phi(s) d s=\int_{-h}^{0} d_{\theta} H(t, \theta) \phi(\theta),\right. \\
\text { Where, } 0 \leq w_{n} \leq h \text { and }\left|\int_{-h}^{0} d_{\theta} H(t, \theta) \phi(\theta)\right| \leq h(\theta)\|\phi\| . \\
\left.D\left(t, x_{t}\right) \text { is non - atomic at zero (i.e differentiable and integrable at zero }\right) . \\
\int_{-h}^{0} A(t, s) d s+\sum_{n-1}^{\infty}\left|A_{n}(t)\right| \leq \delta(\varepsilon) \text {, for all } t, \text { Where }, \delta(\varepsilon) \rightarrow 0 .
\end{gathered}
$$

$f$ is continuous and satisfies other smoothness conditions.

\section{1. Linearization of the System (2.1)}

Consider system (2.1) below

$$
\begin{aligned}
\frac{d}{d t}\left[D\left(t, x_{t}\right)\right]=L\left(t, x_{t}\right) x_{t} & +\int_{0}^{\infty} A(t) x(t+\theta) d \theta+\int_{-h}^{0}\left[d_{\theta} H(t, \theta)\right] u(t+\theta) \\
& +f\left(t, x_{t}\right)
\end{aligned}
$$

(Circularity of the function from $-\infty$ to 0 , and from 0 to $\infty$ ).

We can linearize the system (2.1) as in Chukwu (1992)by setting $x_{t}=z$ in $L$, a specified function inside the function $L\left(t, x_{t}\right) x_{t}$ to have $L(t, z) x_{t}$ without loss of generality. Thus the system (2.1) becomes

$$
\begin{aligned}
\frac{d}{d t}\left[D\left(t, x_{t}\right)\right]=L(t, z) x_{t}+\int_{0}^{\infty} A(t) & x(t+\theta) d \theta \\
& \quad+\int_{-h}^{0}\left[d_{\theta} H(t, \theta)\right] u(t+\theta)+f\left(t, x_{t}\right)
\end{aligned}
$$

Evidently

$$
\begin{gathered}
L(t, z) x_{t}=\sum_{k=0}^{\infty} A_{k} x\left(t-w_{k}\right)+\int_{-\infty}^{0} A(t) x(t+\theta) d \theta \\
\quad+\int_{0}^{\infty} A(t) x(t+\theta) d \theta \\
L^{*}(t, z) x_{t}=\sum_{k=0}^{\infty} A_{k} x\left(t-w_{k}\right)+\int_{-\infty}^{\infty} A(t, \theta) x(t+\theta) d \theta
\end{gathered}
$$

The representation $L, L^{*}$ are the same under the following assumptions.

$$
L(t, z) x_{t}=\operatorname{limit}_{p \rightarrow \infty} \sum_{k=0}^{p} A_{k} x\left(t-w_{k}\right)+\operatorname{limit}_{M . N \rightarrow \infty} \int_{M}^{N} A(t, \theta) x(t+\theta) d \theta
$$

We assume the limits exist, for the partial sum of the infinite series and the improper integrals. Thus the system 


$$
L^{*}(t, z) x_{t}=\sum_{k=0}^{\infty} A_{k} x\left(t-w_{k}\right)+\int_{-\infty}^{\infty} A(t, \theta) x(t+\theta) d \theta
$$

is finite and well defined function.

In the light of the above, the system (2.1) reduces to

$$
\frac{d}{d t}\left[D\left(t, x_{t}\right)\right]=L(t, z) x_{t}+\int_{-h}^{0}\left[d_{\theta} H(t, \theta) u(t+\theta)+f\left(t, x_{t}\right)\right.
$$

$x\left(t_{0}\right)=\phi \in C$.

Where,$L(t, z) x_{t}=\sum_{k=0}^{p} A_{k} x\left(t-w_{k}\right)+\int_{-h}^{0} A(t, \theta) x(t+\theta) d \theta$

\section{2, 2. Variation of Constant Formula}

Integrating system (2.3), after linearizing, we have

$$
\begin{gathered}
x(t)=x\left(t, t_{0}, \phi, u\right)+\int_{0}^{\mathrm{t}} \mathrm{X}(t, s)\left\{\int_{-h}^{0}\left[d_{\theta} H(t, \theta)\right] u(t+\theta)\right\} d s \\
+\int_{0}^{\mathrm{t}} \mathrm{X}(t, s) f\left(s, x_{s}\right) d s
\end{gathered}
$$

Where $X(t, s)$ is the fundamental matrix of the homogeneous part of the system (2.3). $X(t, s)=1$ (identity matrix); $t=s$.

The 2 nd term in the right hand side of system (2.4)contains the values of the control $u(t)$ for $t<t_{0}$, as well as for $t>t_{0}$.

The values of the control $u(t)$ for $t \in\left[t_{0}-h, t_{0}\right]$ enter into the definition

of the initial complete state $u_{t_{0}}$. To separate them, the 2 nd term of system (2.4)must be transformed by changing the order of integration. Using the unsymmetric Fubini theorem, we have the following equalities:

$x(t)=x\left(t, t_{0}, \phi, u\right)+\int_{0}^{\mathrm{t}} \mathrm{x}(t, s) f\left(s, x_{s}\right) d s$

$$
+\int_{-h}^{0} d_{H_{\theta}} \llbracket \int_{t_{0}+\theta}^{t-\theta} X(t, s-\theta) H(s-\theta, \theta) u(s+\theta-\theta) d s \rrbracket
$$

$\Rightarrow$

$x(t)=x\left(t, t_{0}, \phi, u\right)+\int_{\mathrm{t}_{0}}^{\mathrm{t}} \mathrm{x}(t, s) f\left(s, x_{s}\right) d s$

$$
\begin{gathered}
+\int_{-h}^{0} d_{H_{\theta}}\left[\int_{t_{0}+\theta}^{t_{0}} X(t, s-\theta) H(s-\theta, \theta) u_{0}(s) d s\right] \\
+\int_{-h}^{0} d_{H_{\theta}}\left[\int_{t_{o}}^{t} X(t, s-\theta) H(s-\theta, \theta) u(s) d s\right]
\end{gathered}
$$

Where the symbol $d_{H_{\theta}}$ denotes that the integration is in the Lebesque-Stieltjes sense with respect to the variable $\theta$ in the function $H(t, \theta)$.

Let us introduce the following notations:

$$
\widehat{H}(s, \theta)=\left\{\begin{array}{c}
H(s, \theta), \text { for } s \leq t, \theta \epsilon R \\
0, \quad \text { for } s \geq t, \theta \in R
\end{array}\right.
$$


Hence $x(t)$ can be expressed in the following form:

$$
\begin{gathered}
x(t)=x\left(t, t_{0}, \phi, u\right)+\int_{\mathrm{t}_{0}}^{\mathrm{t}} \mathrm{X}(t, s) f\left(s, x_{s}\right) d s \\
+\int_{-h}^{0} d_{H_{\theta}}\left[\int_{t_{0}+\theta}^{t_{0}} X(t, s-\theta) H(s-\theta, \theta) u_{0}(s) d s\right] \\
+\int_{-h}^{0} d_{H_{\theta}}\left[\int_{t_{0}}^{t} X(t, s-\theta) \widehat{H}(s-\theta, \theta) u(s) d s\right]
\end{gathered}
$$

Using again the unsymmetric Fubini theorem, the equality (2.6)can be rewritten in a more convenient form as follows:

$$
\begin{aligned}
x(t)=x\left(t, t_{0}, \phi, u\right)+\int_{0}^{t} \mathrm{x}(t, s) & f\left(s, x_{s}\right) \\
& +\int_{-h}^{0} d_{H_{\theta}}\left[\int_{t_{0}+\theta}^{t_{0}} X(t, s-\theta) H(s-\theta, \theta) u_{0}(s) d s\right] \\
& +\int_{t_{0}}^{t}\left[\int_{-h}^{0} X(t, s-\theta) d_{H_{\theta}} \widehat{H}(s-\theta, \theta)\right] u(s) d s, t_{0}=0 .
\end{aligned}
$$

Now let us consider the solution $x(t)$ of system (2.1) for $t=t_{1}$, we have

$$
\begin{aligned}
& x\left(t_{1}\right)=x\left(t, t_{0}, \phi, u\right)+\int_{0}^{\mathrm{t}} \mathrm{X}(t, s) f\left(s, x_{s}\right) d s \\
& \quad+\int_{-h}^{0} d_{H_{\theta}}\left[\int_{\theta}^{0} X\left(t_{1}, s-\theta\right) H(s-\theta, \theta) u_{t_{0}} d s\right] \\
& \quad+\int_{0}^{t}\left[\int_{-h}^{0} X(t, s-\theta) d_{H_{\theta}} \widehat{H}(s-\theta, \theta)\right] u(s) d s
\end{aligned}
$$

For brevity,

let $B(t)=x\left(t, t_{0}, \phi, u\right)+\int_{0}^{t} X(t, s) f\left(s, x_{s}\right) d s$,

$\mu(t)=\int_{-h}^{0} d_{H_{\theta}}\left[\int_{\theta}^{0} X(t, s-\theta) H(s-\theta, \theta) u_{0}(s) d s\right]$,

and

$\mathrm{Z}(t)=\left[\int_{-h}^{0} X(t, s-\theta) d_{H_{\theta}} \widehat{H}(s-\theta, \theta)\right]$,

so that

$x\left(t, t_{0}, u\right)=B(t)+\mu(t)+\int_{0}^{t} Z\left(t_{1}, s\right) u(s) d s$.

\section{3. Stability Definitions}

We now define the following:

\section{Definition 2.3.1. (Stability)}

The trivial solution $x=0$ of system (2.1) is stable if for any given $t_{0} \in E$, and a positive number $\varepsilon>0$, there exists $\delta=\delta\left(t_{0}, \varepsilon\right)$ such that $\phi \in B(0, \varepsilon)$ implies that

$$
x_{t}\left(t_{0}, \phi\right) \in B(0, \delta)
$$


for all $t \geq t_{0}, \phi \in C$ and $B(0, r)$ is an open ball centered at 0 , with radius $r$.

\section{Definition 2.3.2 (Uniform stability)}

The trivial solution $x=0$ of system (2.1)is uniform stable if for any $\varepsilon>0$, there exists $\delta=\delta(\varepsilon)$ (independent of $t_{0}$ such that $\phi \in B(0, \varepsilon)$ implies that

$$
x_{t}\left(t_{0}, \phi\right) \in B(0, \delta) \text {, for all } t>t_{0} .
$$

\section{Definition 2.3.3 (Asymptotic stability)}

The trivial solution $x=0$ of system (2.1)is asymptotically stable,

if it is stable such that $\phi \in B(0, \delta)$, implies that $x_{t}\left(t_{0}, \phi\right) \rightarrow 0$, as $t \rightarrow \infty$.

\section{Definition 2.3.4 (Uniform Asymptotic stability)}

The trivial solution $x=0$ of system (2.1)is uniformly asymptotically stable if the system is uniformly stable and for $\phi \in B(0, \delta)$, implies that $x_{t}\left(t_{0}, \phi\right) \rightarrow 0$ as $t \rightarrow \infty$.

The solution $x_{t}\left(t_{0}, \phi\right)$ of system (2.1) is exponentially asymptotically stable it there exist constants $k>0$ and $c>0$ such that the solution satisfies $x_{t_{0}}\left(t_{0}, \phi\right)=0$ and

$$
\left|x_{t}\left(t_{0}, \phi\right)\right| \leq k e^{C\left(t-t_{0}\right)} .
$$

\section{Definition 2.3.5 (Complete state)}

It is known that the complete state of system (2.1) at time $t$ is given by

$$
z(t)=\left\{x(t), u_{t}\right\}
$$

Then the initial complete state of the system (2.1) is given by

$$
z(t)=\left\{x_{0}, u_{t_{0}}\right\}
$$

\subsection{BASIC SET FUNCTIONS AND PROPERTIES}

We shall define the set functions upon which our study hinges.

\section{Definition 2.4. 1. (Reachable set)}

The reachable set of the system (2.1) is given as :

$$
R\left(t_{1}, t_{0}\right)=\left\{\begin{array}{c}
\int_{t_{o}}^{t_{1}}\left[\int_{-h}^{0} X(t, s-\theta) d_{H_{\theta}} \widehat{H}(s-\theta, \theta) u(s)\right] d s: u \in U, \\
\text { where } U=\left\{u \in L_{2}\left(\left[0, t_{1}\right], E^{m}\right):|u| \leq 1,\right.
\end{array}\right\}
$$

\section{Definition 2. 4. 2. (Attainable Set)}

The attainable set for the system (2.1) is given as:

$$
A\left(t_{1}, t_{0}\right)=\left\{x\left(t, x_{0}, u\right): u \in U\right\}, \quad \text { where } U=\left\{u \in L_{2}\left(\left[0, t_{1}\right], E^{m}\right):|u| \leq 1\right\} \text {. }
$$

It is a set of all possible solutions of the system (2.1).

\section{Definition 2. 4. 3. (Target Set)}

The target set of system (2.1) denoted by $G\left(t_{1}, t_{0}\right)$ is given as :

$$
\begin{gathered}
G\left(t_{1}, t_{0}\right)=\left\{x\left(t, x_{0}, u\right): t_{1} \geq T>t_{0}, \text { for fixed } T \text { and } u \in U\right\} \text { where } \\
U=\left\{u \in L_{2}\left(\left[t_{0}, t_{1}\right], E^{m}\right):|u| \leq 1\right\}
\end{gathered}
$$




\section{Definition 2. 4. 4. (Controllability grammian)}

The controllability grammian is given as

$$
W\left(t_{1}, t_{0}\right)=\int_{t_{0}}^{t_{1}} Z(t, s) Z^{T}(t, s) d s
$$

$=\int_{t_{0}}^{t_{1}}\left[\int_{-h}^{0} X\left(t_{1}, s-\theta\right) d_{H_{\theta}} \widehat{H}(s-\theta, \theta)\right]\left[\int_{-h}^{0} X\left(t_{1}, s-\theta\right) d_{H_{\theta}} \widehat{H}(s-\theta, \theta)\right]^{T}$

Where $T$ denotes matrix transpose, and, $\quad Z(\boldsymbol{t}, \boldsymbol{s})=\int_{-\boldsymbol{h}}^{\mathbf{0}} \boldsymbol{x}\left(\boldsymbol{t}_{\mathbf{1}}, \boldsymbol{s}-\boldsymbol{\theta}\right) \boldsymbol{d}_{\boldsymbol{H}_{\boldsymbol{\theta}}} \widehat{\boldsymbol{H}}(\boldsymbol{s}-\boldsymbol{\theta}, \boldsymbol{\theta})$.

\subsection{Relationship Between the Set Functions}

We shall first establish the relationship between the attainable set and the reachable set to enable us see that once a property has been proved for one set, then it is applicable to

the other.

From equation (2.8),

$A\left(t_{1}, t_{0}\right)=\left\{\eta(t)+R\left(t_{1}, t_{0}\right)\right\}$ for $u \in U, t \in\left[t_{0}, t_{1}\right]$, Where $\eta(t)=\beta(t)+\mu(t)$.

This means that the attainable set is the translation of the reachable set through $\eta \in E^{n}$. Using the attainable set, therefore, it is easy to show that the set functions possess the properties of convexity, closeness and compactness. Also, the set functions are continuous on $[0, \infty]$ to the metric space of compact subsets of $E^{n}$, (see Chukwu (1988); Gyori (1982). This gives impetus for adaptations of the proofs of these properties for system (2.1).

\subsection{Controllability Standards}

Applications will be made of the following controllability conditions (controllability standard) to establish results:

1.The controllability Grammian or map $W\left(t_{1}, t_{0}\right)$ is invertible and the invertibility of the grammian means that the rank of the grammian must be equal to $n$. i.e Rank $W\left(t_{1}, t_{0}\right)=n \Rightarrow W^{-1}\left(t_{1}, t_{0}\right)$ exists.

2. The nonemptiness of the intersection of two set functions, attainable set $A\left(t_{1}, t_{0}\right)$ and targetset $G\left(t_{1}, t_{0}\right)$ is equivalent to the controllability of the system of interest. That is,

$$
A\left(t_{1}, t_{0}\right) \cap G\left(t_{1}, t_{0}\right) \neq \phi,
$$

implies that the system of interest is controllable.

3. Zero in the interior of the reachable set implies that system of interest is controllable. That is

$$
0 \in \operatorname{Int} A\left(t_{1}, t_{0}\right), \Longrightarrow \text { controllability of the system. }
$$

4.The system (2.1)is proper in $E^{n}$ on $\left[t_{0}, t_{1}\right]$, if $\operatorname{span} R\left(t_{1}, t_{0}\right)=E^{n}$. Or the system (2.1) is proper (controllable) if the controllable index

$$
\begin{gathered}
\mathrm{C}^{\mathrm{T}} \mathrm{Z}(\mathrm{t}, \mathrm{s})=0 \Rightarrow \mathrm{C}=0 \\
\mathrm{C}^{\mathrm{T}}\left[\int_{-\mathrm{h}}^{0} \mathrm{x}\left(\mathrm{t}_{1}, \mathrm{~s}-\theta\right) \mathrm{d}_{\mathrm{H}_{\theta}} \widehat{H}(s-\theta, \theta)\right]=0 \text { a.e }, \boldsymbol{C}=\mathbf{0}, \boldsymbol{C} \in \boldsymbol{E}^{\boldsymbol{n}}, \boldsymbol{t}_{\mathbf{1}}>\mathbf{0} .
\end{gathered}
$$

5. System (2.1) is relatively controllable on $\left[t_{0}, t_{1}\right]$ if, 


$$
A\left(t_{1}, t_{0}\right) \cap G\left(t_{1}, t_{0}\right) \neq \phi ; t_{1}>t_{0} .
$$

\section{MAIN RESULT}

Here, a new method of approach is derived for the proof of the existence of optimal control of our system of interest.

\section{Theorem 3. 1. (Necessary Condition)}

The following are equivalent for system (2.1) visa - via system (1.1) to be controllable:

(1). $W\left(t_{1}, t_{0}\right)$ is non - singular for each $t \in\left[t_{0}, t_{1}\right]$.

(2). The system (2.1) visa - via system(1.1) is proper in $E^{n}$ for each interval $\left[t_{0}, t_{1}\right]$,

(3).The system (2.1)visa - via system(1.1) is relatively controllable on each interval $\left[t_{0}, t_{1}\right]$

\section{Proof}

Recall that $\omega\left(t_{1}, t_{0}\right)$ is non - singular is equivalent to saying that $w\left(t_{1}, t_{0}\right)$ is positive definite, which in turn is equivalent to

$$
C^{T} \int_{t_{0}}^{t_{1}}\left[\int_{-h}^{0} X(t, s-\theta) d_{H_{\theta}} \widehat{H}(s-\theta, \theta)\right] d s=0 \text { a.e on }\left[t_{0}, t_{1}\right], \text { implies } C=0 .
$$

Therefore, showing that (1) and (2) are equivalent.

To show that (2) and (3) are equivalent:

$$
\begin{gathered}
C^{T} \int_{t_{0}}^{t_{1}}\left[\int_{-h}^{0} X(t, s-\theta) d_{H_{\theta}} \widehat{H}(s-\theta, \theta)\right] d s=0 \text { a.e. } t \in\left[t_{0}, t_{1}\right] \text {, for each } t, \text { then } \\
C^{T} \int_{t_{0}}^{t_{1}}\left[\int_{-h}^{0} X(t, s-\theta) d_{H_{\theta}} \widehat{H}(s-\theta, \theta)\right] u(s) d s \\
=\int_{t_{0}}^{t_{1}} C^{T}\left[\int_{-h}^{0} X(t, s-\theta) d_{H_{\theta}} \widehat{H}(s-\theta, \theta)\right] u(s) d s=0, \text { for } u \in L_{2}
\end{gathered}
$$

It follows from this that $C$ is orthogonal to the reachable set

$$
R\left(t_{1}, t_{0,}\right)=\left\{\int_{t_{0}}^{t_{1}}\left[\int_{-h}^{0} X(t, s-\theta) d_{H_{\theta}} \widehat{H}(s-\theta, \theta)\right] u(s) d s ;|u| \leq 1\right\}
$$

If we assume the relative controllability of system (2.1) now, then, $R\left(t_{1}, t_{0}\right)=E^{n}$,

so that $C=0, \quad$ showing that (3) implies (2).

Conversely, assume for contradiction that system (2.1)is not controllable so that $R\left(t_{1}, t_{0}\right) \neq E^{n}$, for $t_{1}>t_{0}$.Then, there exists $C \neq 0, C \in E^{n}$, such that $C^{T} R\left(t_{1}, t_{0}\right)=0$. It now follows that for all admissible controls $u \in L_{2}$ that

$$
0=C^{T} \int_{t_{0}}^{t_{1}}\left[\int_{-h}^{0} X(t, s-\theta) d_{H_{\theta}} \widehat{H}(s-\theta, \theta)\right] u(s) d s
$$




$$
=\int_{t_{0}}^{t_{1}} C^{T}\left[\int_{-h}^{0} x(t, s-\theta) d_{H_{\theta}} \widehat{H}(s-\theta, \theta)\right] u(s) d s
$$

Hence,

$$
C^{T} \int_{t_{0}}^{t_{1}}\left[\int_{-h}^{0} x(t, s-\theta) d_{H_{\theta}} \widehat{H}(s-\theta, \theta)\right] u(s) d s=0 \quad \text { a.e }, s \in\left[t_{0}, t_{1}\right], C \neq 0 .
$$

This, by definition of properness implies the system is not proper, since $C^{T} \neq 0$. Hence the system is relatively controllable.

\section{Theorem 3.2}

Consider system(2.1)visa - via system (1.1)

$$
\frac{d}{d t}[D(t, x)]=L(t, x \mathrm{t}) x_{t}+\int_{0}^{\infty} A(t, \theta) x(t+\theta) d \theta+\int_{-h}^{0}\left[d_{\theta} H(t, \theta)\right] u(t+\theta)+f\left(t, x_{t}\right)
$$

with its standing hypothesis. Suppose that $u^{*}$ is the optimal control, then it is unique.

\section{Proof}

Let $u^{*}$ and $v^{*}$ be optimal controls for the system (3.1), then $u^{*}$ and $v^{*}$ maximize

$$
C^{T} \int_{t_{o}}^{t_{1}}\left[\int_{-\mathrm{h}}^{0} \mathrm{X}(\mathrm{t}, \mathrm{s}-\theta) \mathrm{d}_{\mathrm{H}_{\theta}} \widehat{H}(s-\theta, \theta)\right] u(s) d s, \text { for } t \in\left[0, t_{1}\right] ; t_{1}>0 \text {, }
$$

Over all admissible controls $u \in U$, and also we have the inequality with $u^{*}$ as the optimal control.

$$
\begin{aligned}
& C^{T} \int_{0}^{t}\left[\int_{-\mathrm{h}}^{0} \mathrm{X}(\mathrm{t}, \mathrm{s}-\theta) \mathrm{d}_{\mathrm{H}_{\theta}} \widehat{H}(s-\theta, \theta)\right] u(s) d s \\
& \leq \int_{0}^{t} C^{T}\left[\int_{-\mathrm{h}}^{0} \mathrm{X}(\mathrm{t}, \mathrm{s}-\theta) \mathrm{d}_{\mathrm{H}_{\theta}} \widehat{H}(s-\theta, \theta)\right] u^{*}(s) d s
\end{aligned}
$$

Also, using $v^{*}$ as optimal control, we have

$$
\begin{aligned}
C^{T} \int_{0}^{t}\left[\int_{-\mathrm{h}}^{0} \mathrm{X}(\mathrm{t}, \mathrm{s}-\theta) \mathrm{d}_{\mathrm{H}_{\theta}} \widehat{H}(s-\theta, \theta)\right] u(s) d s & \\
& \leq \int_{0}^{t^{*}} C^{T}\left[\int_{-\mathrm{h}}^{0} \mathrm{X}(\mathrm{t}, \mathrm{s}-\theta) \mathrm{d}_{\mathrm{H}_{\theta}} \widehat{H}(s-\theta, \theta)\right] v^{*}(s) d s
\end{aligned}
$$

Taking maximum of $u$, over $[-1,1]$, the range of definition of $u^{*}$ in system (3.2) and (3.3), we have $C^{T} \int_{0}^{t}\left[\int_{-\mathrm{h}}^{0} \mathrm{X}(\mathrm{t}, \mathrm{s}-\theta) \mathrm{d}_{\mathrm{H}_{\theta}} \widehat{H}(s-\theta, \theta)\right] \max |u(s)| d s$;

$$
=C^{T} \int_{0}^{t}\left[\int_{-\mathrm{h}}^{0} \mathrm{X}(\mathrm{t}, \mathrm{s}-\theta) \mathrm{d}_{\mathrm{H}_{\theta}} \widehat{H}(s-\theta, \theta)\right] u^{*}(s) d s, \quad \text { for } u, u^{*} \in U \text {. }
$$

Also,

$$
\begin{aligned}
C^{T} \int_{0}^{t}\left[\int_{-\mathrm{h}}^{0} \mathrm{X}(\mathrm{t}, \mathrm{s}-\theta)\right. & \left.\mathrm{d}_{\mathrm{H}_{\theta}} \widehat{H}(s-\theta, \theta)\right] \max |u(s)| d s ; \\
& =C^{T} \int_{0}^{t}\left[\int_{-\mathrm{h}}^{0} \mathrm{X}(\mathrm{t}, \mathrm{s}-\theta) \mathrm{d}_{\mathrm{H}_{\theta}} \widehat{H}(s-\theta, \theta)\right] v^{*}(s) d s, \quad \text { for } u, v^{*} \in U .
\end{aligned}
$$

$v^{*}(s)$ being optimal control such that $-1 \leq s \leq 1$. 


$$
\begin{gathered}
C^{T} \int_{0}^{t}\left[\int_{-\mathrm{h}}^{0} \mathrm{X}(\mathrm{t}, \mathrm{s}-\theta) \mathrm{d}_{\mathrm{H}_{\theta}} \widehat{H}(s-\theta, \theta)\right] \max |u(s)| d s \\
=0 \quad C^{T} \int_{0}^{t}\left[\int_{-\mathrm{h}}^{0} \mathrm{X}(\mathrm{t}, \mathrm{s}-\theta) \mathrm{d}_{\mathrm{H}_{\theta}} \widehat{H}(s-\theta, \theta)\right] \max |u(s)| d s \\
=C^{T} \int_{0}^{t}\left[\int_{-\mathrm{h}}^{0} \mathrm{X}(\mathrm{t}, \mathrm{s}-\theta) \mathrm{d}_{\mathrm{H}_{\theta}} \widehat{H}(s-\theta, \theta)\right] u^{*}(s) d s, \\
\Rightarrow 0=c^{T} \int_{0}^{t}\left[\int_{-\mathrm{h}}^{t}\left[\int_{-\mathrm{h}}^{0} \mathrm{X}(\mathrm{t}, \mathrm{s}-\theta) \mathrm{d}_{\mathrm{H}_{\theta}} \widehat{H}(s-\theta) \mathrm{d}_{\mathrm{H}_{\theta}} \widehat{H}(s-\theta)\right]\left\{u^{*}(s)-v^{*}(s)\right\} d s=\right. \\
\Rightarrow u^{*}(s)-v^{*}(s)=0 \\
\Rightarrow u^{*}(s)=v^{*}(s) .
\end{gathered}
$$

This establishes the uniqueness of the optimal control for the system (3.1) visa via system (1.1).

\section{EXAMPLE}

\section{Example4.1}

Consider the control linear system given below:

$\dot{x}(t)=A x(t)+B u(t)$

where $A=\left[\begin{array}{ll}0 & 1 \\ 0 & 0\end{array}\right]$ and $B=\left[\begin{array}{l}0 \\ 1\end{array}\right] ; x(0)=\left(x_{0}, y_{0}\right)$, where $U=\left\{u \in L_{2}\left(\left[0, t_{1}\right], E^{m}\right):|u| \leq 1\right\}$.

Does the system (1) has an optimal control whic is unique?

\section{Solution}

$A=\left[\begin{array}{ll}0 & 1 \\ 0 & 0\end{array}\right]$ and $B=\left[\begin{array}{l}0 \\ 1\end{array}\right]$.

It is easy to verify that the fundamental matrix $X(t)=e^{A t}=\left[\begin{array}{ll}1 & t \\ 0 & 1\end{array}\right]$ and

$X^{-1}(t)=e^{-A t}=\left[\begin{array}{ll}1 & t \\ 0 & 1\end{array}\right]$.

Question: Is the system(1) controllable? If yes, then there exists an optimal control

Answer : For controllability,

$\operatorname{rank}[B, A B]=\operatorname{rank}\left[\left[\begin{array}{l}0 \\ 1\end{array}\right],\left[\begin{array}{ll}0 & 1 \\ 0 & 0\end{array}\right]\left[\begin{array}{l}0 \\ 1\end{array}\right]\right]=\operatorname{rank}\left[\left[\begin{array}{l}0 \\ 1\end{array}\right],\left[\begin{array}{l}1 \\ 0\end{array}\right]\right]=\operatorname{rank}\left[\begin{array}{ll}0 & 1 \\ 1 & 0\end{array}\right]=2=n$

Therefore, the system(1) is proper and hence controllable. The system(1) is also normal since B is a column matrix.Thus, the optimal control will be unique and bang - bang and of the form

$$
\begin{gathered}
u^{*}=\operatorname{sgnC}^{T} X^{-1}(t) B(t)=\operatorname{sgn}\left[C e^{-A t} B(t)\right] \\
=\operatorname{sgn}\left[\left(\begin{array}{ll}
C_{1} & C_{2}
\end{array}\right)\left(\begin{array}{cc}
1 & -t \\
0 & 1
\end{array}\right)\left(\begin{array}{l}
0 \\
1
\end{array}\right)\right]=\operatorname{sgn}\left[\left(\begin{array}{ll}
C_{1} & -C_{1} t+C_{2}
\end{array}\right)\left(\begin{array}{l}
0 \\
1
\end{array}\right)\right] \\
=\operatorname{sgn}\left[\begin{array}{ll}
0 & -C_{1} t+C_{2}
\end{array}\right]=\operatorname{sgn}\left(-C_{1} t+C_{2}\right), \quad \text { for some }\left(\begin{array}{ll}
C_{1} & C_{2}
\end{array}\right) \in E^{n} .
\end{gathered}
$$

Hence $u^{*}$ has only one switch between -1 and 1 .

To see this, we solve

$$
-C_{1} t+C_{2}=0, \Rightarrow-C_{1} t=-C_{2}, \Rightarrow t=\frac{C_{2}}{C_{1}} \text { which gives value of } t \text {. }
$$




\section{CONCLUSION}

In this work, therefore, we have established necessary and sufficient conditions for the system (1.1) to have an optimal control .by establishing the controllability of the system. The optimal control of the system (1.1) was, and then proved to be unique. Thus, the uniqueness of the optimal of the system (1.1) was established.

Not alone, the processes of the establishment of the uniqueness of the optimal control provided a new approach to the establishment of the existence of optimal control of any dynamical system.

\section{REFERENCES}

Anichini, G. Conti and Zecca, P. (1986),"A Note on Controllability of Certain Nonlinear Systems”, Note the Math., Vol.6, Pp 1, 99 - 111. Artstein, Z. (1982), "Linear Systems with Delayed Control, - A Reduction”, I.E.E.E. Transations om Automatic Control, Ac - 27, Pp 869 879.

Balachandran, K. (1992), “Controllability of Neutral Volterva Integro-differential Systems”, Journal of Australian Mathematical Society Ser B34, Pp 18-25.

Balanchandran, K. and J.P Dauer (1996), "Local Null Controllability of Nonlinear Functional Differential System in Banach Spaces” Journal of optimal theory and App. 88, pp 61-75.

Balanchandran, K. and J.P Dauer (2002), “Controllability of Nonlinear Systems in Banach Spaces”, A Survey Jota 115.

Balanchandran, K. andn A. Leelamani (2006), "Existence Results for Nonlinear Abstract Neutral Differential Equations with Time Varying Delays”, Applied Mathematics Noyes 6, Pp $186-193$.

Banks, H.T., and Kent G.A., (1972), "Control of Functional Differential Equations of Retarded and Neutral Type to Target sets in Function Spaces” S.I.A.M., Journal on Control 10, Pp 562-593.

Chukwu E. N. (1988), "The Time Optimal Control Theory of Linear Differential Equations of Neutral Type", Journal Computer. Maths and Applications, vol.16 pp 851-866.

Chukwu, E.N. (1992), "Stability and Time Optimal Control of Hereditary Systems,” Academic Press, New York.

Chukwu, E.N. (2001), "Differential Models and Neutral Systems for Controlling the Wealth of Nations" Services on Advance in Mathematics from Applied Sciences, Vol.54. World Scientific, New Jersey.

Gyori, I. (1982), "Delay Differential and Integrodifferential Equations in Biological Compartmental Models" Systems Science (Wroclaw) Poland, 8,pp 7-187.

Onwuatu, J.U. (1993), "Null Controllability of Nonlinear Infinite Neutral Systems”, Kyberuetika, Vol.29, Pp 1-12.

\section{$\underline{\text { How to cite this article }}$}

Oraekie, P.A. (2018). Uniqueness of Optimal Control of Nonlinear Infinite Neutral Functional Differential Systems with Distributed Delays in the Control. Tropical Journal of Applied Natural Sciences, 2(2): 15-25. Doi: https://doi.org/10.25240/TJANS.2018.2.2.03. 